Monatsschrift f. Geburtshülfe u. Gynäkologie 1937;106:I-IV

\title{
Contents, Vol. 106, 1937
}

Inhaltsverzeichnis.

Originalarbeiten. seite

Bonilla, Francisco, und Kramann, Heinrich, Über thyreotropes Mor

mon in der Schwang erschaît. II. Mitteilung 24

Föderl, Viktor, 3/8ur Kenntnis des Arrhenoblastoms 54

Günther, Die Prokteuryse (rektale Tokokinese) und ihr heutiger Wert

neben den medikamentösen Wehenmitteln 138

Hildebrandt, Alwín, Vergleiehende Untersuehung en mit der Schwangerschaftsreaktion nach Visscher und Bowman sowie mit ihren

Modifikationen 129

Hollósi, Karl, Appendizitis und Menstruation 187

Kochs, Albert G., Untersuchungen über die Physiologic der Brust-

drüse 1

Kramann, Heinrich, siehe Bonilla, Francisco.

Kramann, Heinrich, Über ein malignes, ein Ovarialkystom vortäu-

schendes 3Iyom des Ileum 27

Kriss, Bruno, Feminisierende Wirkung des männliehen Sexual-

hormons

157

Leinzinger, E., Ein Todesfall durch toxischen Geburtssehoek ... 331 Martius, H., Welchen Wert hat die Böntg endiagnostik bei der Geburt

beim engen Beckenï 257

Mondt, W., Die Erfolge exspektativer Abortbehandlung · 291

Neukamp, Franz, Berufsverbot gegen emeu wegen Abtreibung bestratten Arzt 304

Ramos, Alberto Peralta, und Uranga-Imaz, Francisco A., Zvvei

gleichzeitige Sehwangerschaïten in einem Uterus duplex . . 178 Rauscher, H., Über Hornbildung in einem Ovarialkarziiiom . . . 149 Reiber, H., Behandlung · fieberhafter Aborte in den letzten drei Jahren 298 Reifferscheid, W., Über die Jokale Behandlung· des Pruritus vulvae mit Follikelhormon 287

Schlesinger, Herbert, Hautemphysem nach Sectio caesarea 66

Schwalm, H., Die Erythrozyten-Eegeneration nach g·ynäkologischen

und geburtshilflichen Blutungen (mit Untersuchungen über den

Einfluß des Follikelhormons auf die Erythropoese) 274

J"y Inhaltsverzeichnis.

Seiie

Sehwalm, II., Dauerîolgen am > veiblichen Genitale nach früheren

Entzündungen im Beckenraum 343 
Steiner, Peter, Todesîälle nach gynäkoiogîschen Operationen ... 33 Síepowski, Bronislaw, Über die Wirkung der Sexualhormone auï den künstlich hervorgerufenen Reîiungsprozcß der Graafsehen Follikel $\quad 167$

Szczepanski, Walter, Wehen und Headsche Zonen 325

Tausch, AL, Über interkurrente Eklampsie 321

Uranga-Imaz, Francisco A., siehe Ramos, Alberto Peralta.

Grenzgebiete der Geburtshilie und Gynäkologie.

Soziale Gynäkologie und Geburtshilfe. $\Lambda \tau$ on Dozent Dr. R. Hof-

stätter-Wien 77

Physiologie und Krankheiten der Neugeborenen (Juli 1935-1937).

Von Hans RuscL - - Gießcn 193

ïuberkulosc als Grenzgebiet der Geburtshilîe und Gynäkologie. Von

Dr. V. Probst-Breslau 3()(í

Zur Trage der Sehmerzbekämpiung. Von Dr. Franz Hoí'f-Würzburg 351

Sitzungsberichte aus geburtshilflich - gynäkoiogîschen Gesellschaften.

Geburtshilflich-Gymikoloí > isehe Gesellsehaft in Wien. Sitzung vom

13. April 1937252

Gesellsehaft für Geburtshilfe und Gynäkologie zu Berlin. Sitzung

vom 15. Januar 1937313

GynäkoJogische Gesellsehaft zu Breslau. Sitzung vom 17. Januar 1936316

98. Sitzung der Mittelrhein. Gesellsehaft für Geburtshilfe und Gynä

kologie am 21. Februar 1937 in Frankfurt a. M 366

Einladung < ler Dcutseheti Gesellsehaft für Gynäkologie 253

Tagesnael $\pi$-iehteii $\quad 256$

Buchbespreehnngen 319 